\title{
Comparison of safety and efficacy of $20 \%$ salicylic acid with Jessner's solution in chemical peeling for melasma in asian skin
}

\author{
Uzma Ahsan', Muhammad Uzair², Humaira Aamir ${ }^{1}$ \\ ${ }^{1}$ Sharif Medical City, Lahore, Pakistan, ${ }^{2} \mathrm{KMU}-\mathrm{IMS}$, Kohat, Pakistan \\ Corresponding author: Dr. Muhammad Uzair, E-mail: waziruzair1@gmail.com
}

\begin{abstract}
Background: Treatment of melasma is a challenging job for dematologists. Different modalities are in practice with variable results. Superficial chemical peeling is a newly introduced way of treatment in this regard, in which a chemical solution is applied to the skin that causes exfoliation and peeling. Methods: Sixty patients of either sex, with melasma of any type having Fitzpatrick skin type 4 to 6 were enroled from outpatient department. Patients in group A were treated with $20 \%$ salicylic acid solution and in group B were treated with Jessener's solution. Baseline melasma area and severity index (MASI) scoring was done. Result: Both the peeling agents produced a drop in MASI score, but the mean reduction compared in both the groups was not statistically significant ( $\mathrm{p}>0.05)$. Conclusion: Both salicylic acid and Jessner's solution are effective and safe peeling agents in treatment of melasma in Asian skin types, giving comparable results
\end{abstract}

Key words: Melasma; Salicylic acid; Jessner’s solution

\section{INTRODUCTION}

Treatment of Melasma is a common aesthetic fray in dermatology. Various topical depigmenting agents have been used alone or in combination with varying results but no agent has proved to be ideal. Superficial chemical peeling is a newly introduced modality in this regard [1].

A chemical peel is a method used to improve the appearance of the skin on the face, neck or hands. A chemical solution is applied to the skin that causes exfoliation and peeling. The new, rejuvenated skin is usually gentle and less wrinkled than the old skin. The new skin is also temporarily more photosensitive [2]. Chemical peels are popular aesthetic procedures in America and superficial chemical peels have recently been demonstrated to be efficacious in Asian skin as well in various conditions [3].
Salicylic acid is among the most promising superficial chemical peeling agents used in epidermal melasma. It possesses keratolytic, comedolytic and antiinflammatory properties. It solubilizes intercellular bond, reduces corneocytes adhesion, and sloughs off the superficial layers of skin $[4,5]$.

Despite plenty of articles on the use of alpha hydroxy acids as peeling agents in literature, there is a paucity of published data regarding the efficacy and safety of salicylic acid peels in Asian skin.

Jessner's peel is an intermixture of salicylic acid $14 \%$, lactic acid $14 \%$, and resorcinol $14 \%$ in alcohol. Jessner's solution has a historic use of over 100 years. Conventionally, it has been used as medium depth chemical peel. Recently, it emerged as superficial chemical peeling agent and studies are required to testify its role as effective superficial peel [6].

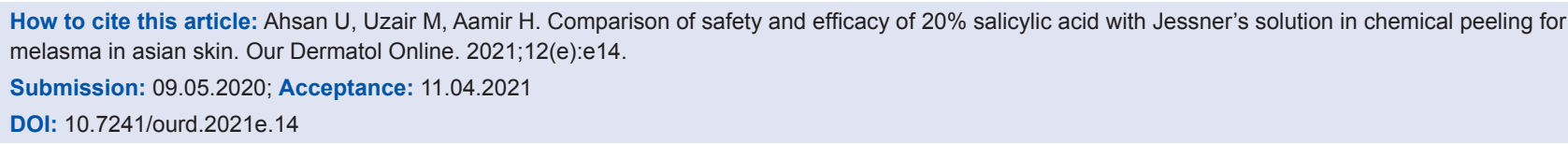


Literature review highlights the efficacy of both these agents but the data to compare the efficacy is sparse. So it was decided to compare the efficacy of two chemical peeling agents, Jessner's solution with $20 \%$ salicylic acid in the treatment of melasma.

\section{MATERIALS AND METHODS}

The study was conducted in the Department of Dermatology, Sharif Medical \& Dental College. A total of 60 patients were enrolled in the study. The sample size was calculated using WHO calculator for two groups for a difference in proportion of $20 \%$ between the groups giving significance of 0.05 and power of $80 \%$. It was a quasi experimental study started after approval of ethical review board of the hospital.

60 patients of either sex with melasma of any type (epidermal, dermal, and mixed) as assessed by Wood's lamp examination, having Fitzpatrick's skin type IV and $\mathrm{V}$ were included in the study. They were enrolled from outpatients department. A written consent was taken from the patients. Before, during and after treatment photographs of the patients were also taken after informed and written consent.

Patients unable to avoid excessive sun exposure, pregnant women, lactating women, patients with liver disease, patients using contraception \& hormonal therapy and patients already taking topical or systemic medicine for melasma were excluded. All selected patients were clearly advised not to apply cosmetics or any other topical application during the study period. Patients were randomly assigned to either group A or group B using computer generated randomization software. Patients in group A were treated with 20\% salicylic acid solution and in group B were treated with Jessener's solution. Patients were advised to use tretinoin $0.05 \%$ cream at night for two weeks (stopped 1 week before the procedure) as pre-peel priming. Baseline melasma area and severity index (MASI) scoring was done. Freshly prepared Jessener's solution kept in amber colored bottle was used keeping in view the issue of its rapid degeneration. Night-time use of moisturizer was prescribed in all patients. At each visit, compliance was reassured. Patients were blinded to the peeling agent being used on them but it was not possible to blind the investigator due to different colors of the peeling agents. However, the investigator responsible for Melasma Area Severity Index (MASI) scoring was blinded to the treatment. Baseline MASI score was noted and patients were called at 2 weekly intervals for chemical peeling. The face was first cleansed with soap and water then dried with cotton swab. A layer of chemical peel was then applied with a cotton swab to enable distribution of peeling solution. Using cotton tipped sticks; the assigned solution was applied in a thin layer till even frosting occurred. The solution was left for 10 minutes and then washed with water. The patients were advised strict sun protection with use of sun block cream before venturing outdoors. The duration of action of sunscreen was fully explained to the patients. Patients were called after every 2 weeks to document the MASI score for assessment and repeat procedure. At the end of 12 weeks, procedure was stopped and final MASI score was noted. At the end of peeling sessions, patients had a follow up examination at 4 weeks after the last session to assess the effectiveness of the peeling agent on a 4-grade scale. This scale divided the patients into following categories. Excellent (>75\% improvement), Good (50-75\% improvement), Fair (25-49\% improvement Poor) (<25\%improvement). Safety was established by assessing the side effects and observing the degree of tolerability to the peel.

\section{Ethics Statement}

The study was approved by the ethical committee of the institution

\section{RESULTS}

The statistical analysis was done using computer programme SPSS version 21.0. Frequencies and percentages for the variables were reported. Significance was tested at a level of 0.05 using chi- square test. Out of 60 patients, who entered the study, 8 (11.8\%) were males and $52(88.2 \%)$ were females. Age ranged from 20 to 44 years (mean 30.4 years, SD + 5.8). $42(70 \%)$ patients had type IV skin and 18 (30\%) were with type V skin. 29 (48\%) patients had mixed type of melasma, $26(43 \%)$ patients presented with epidermal and only $5(8 \%)$ had dermal melasma.

Group A (20\% salicylic acid) included 34 (56.7\%, mean age 28.1 years) patients, while group B (Jessener's solution) included 26 (43.3\%, mean age 32.4 years) patients. Majority of the patients were housewives who did not give history of excessive sun exposure.

At the end of treatment period, there were $3(6 \%)$ dropouts in group A and 1 (3.8\%) in group B. MASI 
score dropped in both these groups at the end of 12 weeks. Details of pre-peeling and post peeling MASI score is tabulated in Table 1.

Both the peeling agents produced a drop in MASI score, but the mean reduction compared in both the groups was not statistically significant $(\mathrm{p}>0.05)$. Assessment of efficacy using 4-grade analysis score was also done and results were tabulated in Table 2.

We analyzed and compared the efficacy of both the peeling agents with respect to the duration and type of melasma, displayed in Table 3 and 4 respectively.

\section{DISCUSSION}

Melasma is primarily a disease of females. In our study $88.2 \%$ were females compared to males who were $11.2 \%$. This was supported by studies conducted by Sarkar et al. [5], and Lee et al. [7] highlighting female predominance with $\mathrm{M}: \mathrm{F}$ ratio of 1:3. Female preponderance can possibly be due to

Table 1: Comparison of pre-treatment and post treatment MASI Score

\begin{tabular}{lcc}
\hline Group & $\begin{array}{c}\text { Pre Treatment } \\
\text { MASI Score } \\
\text { (mean) }\end{array}$ & $\begin{array}{c}\text { Post Treatment } \\
\text { MASI Score } \\
\text { (mean). }\end{array}$ \\
\hline $\begin{array}{l}\text { Group A } \\
\text { (20\% salicylic acid) }\end{array}$ & $16.54 \pm 5.32$ & $10.5 \pm 2.4$ \\
$\begin{array}{l}\text { Group B (Jessener's } \\
\text { Solution) }\end{array}$ & $15.31 \pm 4.52$ & $9.8 \pm 3.7$ \\
\hline
\end{tabular}

the high incidence of melasma in women. Pregnancy, oral contraceptive pills, and estrogen-progesterone therapies may be the other factors in women contribute to etiopathogenesis of this condition. Other factors for high incidence of melasma in females presenting to outpatient department can be the over consciousness among them regarding cosmetic disfigurement caused by melasma.

Mean age in our study was 30.4 years. This was in comparison with other studies performed by Ejaz A. et al [8] and Bari AU et al [9] and but in disparity to the study done by Vedamurthy $\mathrm{M}$ et al [10], where the mean age was 42.3 years.

The response to the treatment was inversely proportional to the duration of melasma. Our results were consistent with the local $[8,9]$ as well as global data where peeling agents were proven to be more effective in patients with lesser duration of melasma, and those with longer duration were categorized among poor responders $[10,11]$.

Treatment of melasma is challenging, especially in dark skinned individuals [12]. Different modalities are in practice worldwide. In this study Salicylic acid peel was compared with Jessener's solution. MASI Score at the end of peeling sessions was compared with the pre-peeling scores. Both the groups demonstrated a decline in MASI score. Patients in Group A (Treated with salicylic acid) MASI score decreased from a mean

Table 2: Comparison of Efficacy between $20 \%$ Salicylic acid and Jessener's solution

\begin{tabular}{lcccc}
\hline Group & $\begin{array}{c}\text { Excellent (>75\% } \\
\text { improvement) }\end{array}$ & $\begin{array}{c}\text { Good (50-75\% } \\
\text { improvement) }\end{array}$ & $\begin{array}{c}\text { Fair (25-49\% } \\
\text { improvement }\end{array}$ & $\begin{array}{c}\text { Total } \\
\text { (<25\%improvement ) }\end{array}$ \\
\hline 20\%salicylic Acid & 6 & 21 & 5 & 2 \\
(Jessener's Solution) & 4 & 14 & 7 & 24 \\
\hline
\end{tabular}

Table 3: Comparison of Efficacy Between 20\% Salicylic Acid and Jessener's Solution With Respect To Duration of Melasma

\begin{tabular}{llcccc}
\hline Group & Duration & $\begin{array}{c}\text { Excellent (>75\% } \\
\text { improvement) }\end{array}$ & $\begin{array}{c}\text { Good (50-75\% } \\
\text { improvement) }\end{array}$ & $\begin{array}{c}\text { Fair (25-49\% } \\
\text { improvement }\end{array}$ & $\begin{array}{c}\text { Total } \\
\text { (<25\%improvement ) }\end{array}$ \\
\hline \multirow{2}{*}{ 20\%salicylic Acid } & $1-5$ years & 5 & 15 & 2 & 4 \\
Jessener's Solution & $6-10$ years & 1 & 6 & 1 & 26 \\
& $1-5$ years & 3 & 10 & 5 & 2 \\
\hline
\end{tabular}

Table 4: Comparison of Efficacy Between 20\% Salicylic Acid and Jessener's Solution With Respect To clinical Type of Melasma

\begin{tabular}{|c|c|c|c|c|c|c|}
\hline Group & $\begin{array}{l}\text { Type of } \\
\text { Melasma }\end{array}$ & $\begin{array}{c}\text { Excellent (>75\% } \\
\text { improvement) }\end{array}$ & $\begin{array}{l}\text { Good (50-75\% } \\
\text { improvement) }\end{array}$ & $\begin{array}{c}\text { Fair }(25-49 \% \\
\text { improvement }\end{array}$ & $\begin{array}{c}\text { Poor } \\
\text { (<25\%improvement ) }\end{array}$ & Total \\
\hline $20 \%$ salicylic & Dermal & 0 & 2 & 1 & 2 & 5 \\
\hline \multirow[t]{2}{*}{ Acid } & Epidermal & 4 & 18 & 2 & 0 & 24 \\
\hline & Mixed & 2 & 1 & 2 & 0 & 5 \\
\hline Jessener's & Dermal & 0 & 2 & 2 & 0 & 4 \\
\hline \multirow[t]{2}{*}{ Solution } & Epidermal & 2 & 10 & 3 & 1 & 16 \\
\hline & Mixed & 2 & 2 & 2 & 0 & 6 \\
\hline
\end{tabular}


of 16.5 to 10.5 , while those in Group B (Treated with Jessner's Solution) it dropped down in mean 9.3 from a mean of 15.3.The difference when compared was not statistically significant $(\mathrm{P}>0.05)$. These findings were consistent with the results of Ejaz A. et al. [8]. In studies by Godse et al. [13] and Erbil et al. [14], mean MASI score significantly decreased from $19.72 \pm 6.71$ to 10.17 and from 18.67 to 11.30 , respectively. The difference in results could possibly be due to the use of a different peeling agent and also due to the addition of triple combination of tretinoin $0.05 \%$, hydroquinone $4 \%$ and mometasone furoate $0.1 \%$ along with the serial Glycolic Acid peels by Godse et al. [13]. Similarly Erbil et al. [14] used serial Glycolic Acid peels from 35\% to $70 \%$ along with topical application of azelaic acid. This difference in results may be because of a better efficacy of glycolic acid and also by the simultaneous use of topical depigmenting agents in both the studies. Further research is considered necessary to evaluate the efficacy of glycolic acid peels in comparison to salicylic acid and Jessner's solution in melasma.

Comparison of results in reference to the type of melasma revealed a better response in epidermal type of melasma in both groups. Patients in group A showed better results to salicylic acid peel as compared to Jessner's solution in group B. Both salicylic acid and Jessner's solution peels were less effective in mixed type of melasma and with no good results in dermal type except 2 patients. These findings were supported by another local study $[15,16]$.

In our study we used $20 \%$ salicylic acid with almost similar results as compared to Ejaz A. et al, where $30 \%$ salicylic acid was used as a peeling agent, demonstrating that salicylic acid is as effective at low concentration which can result in fewer side effects.

\section{CONCLUSION}

Both salicylic acid and Jessner's solution are effective and safe peeling agents in treatment of melasma in Asian skin types, giving comparable results. Further studies are however needed to compare the efficacy of these agents with other chemical peeling agents and combining these with topical depigmenting agents in Asian skin types.

\section{Statement of Human and Animal Rights}

All procedures followed were in accordance with the ethical standards of the responsible committee on human experimentation (institutional and national) and with the Helsinki Declaration of 1975, as revised in 2008.

\section{Statement of Informed Consent}

Informed consent was obtained from all patients for being included in the study.

\section{REFERENCES}

1. Hassan KM, Benedetto AV. Facial skin rejuvenation: ablative laser resurfacing, chemical peels, or photodynamic therapy? Facts and controversies. Clin Dermatol. 2013;31:737-40.

2. Tao L, Wu J, Qian H, Lu Z, Li Y, Wang W, et al. Intense pulsed light, near infrared pulsed light, and fractional laser combination therapy for skin rejuvenation in Asian subjects: a prospective multi-center study in China. Lasers Med Scien. 2015;30:1977-83.

3. Salam A, Dadzie OE, Galadari H. Chemical peeling in ethnic skin: an update. Br J Dermatol. 2013;169:82-90.

4. Lage R, Mendes C, Zugaib BM, Abdalla JA, Costa A. Cosmeceutical Ingredients: Botanical and Nonbotanical Sources. Daily Rout Cosmet Dermatol. 2017:203.

5. Sarkar R, Bansal S, Garg VK. Chemical peels for melasma in darkskinned patients. J Cutan Aesthet Surg. 2012;5:247-53.

6. Soleymani T, Lanoue J, Rahman Z. A practical approach to chemical peels: a review of fundamentals and step-by-step algorithmic protocol for treatment. J Clin Aesthet Dermatol. 2018;11:21.

7. Lee AY. An updated review of melasma pathogenesis. Dermatol Sinica. 2014;32:233-9.

8. Ejaz A, Raza N, Iftikhar N, Muzzafar F. Comparison of $30 \%$ salicylic acid with Jessener's Solution for superficial chemical peeling in epidermal melasma. J Coll Physicians Surg Pak. 2008;18:205-8.

9. Saleh F, Moftah NH, Abdel-Azim E, Gharieb MG. Q-switched Nd: YAG laser alone or with modified Jessner chemical peeling for treatment of mixed melasma in dark skin types: A comparative clinical, histopathological, and immunohistochemical study. J Cosmet Dermatol. 2018;17:319-27.

10. Rivas S, Pandya AG. Treatment of melasma with topical agents, peels and lasers: an evidence-based review. Am J Clin Dermatol. 2013;14:359-76..

11. Sarma N, Chakraborty S, Poojary SA, Rathi S, Kumaran S, Nirmal B, et al. Evidence-based review, grade of recommendation, and suggested treatment recommendations for melasma. Indian Dermatol Online J. 2017;8:406.

12. Sarkar R, Bansal S, Garg VK. Chemical peels for melasma in darkskinned patients. J Cutan Aesthet Surg. 2012;5:247.

13. Godse KV, Sakhia J. Triple combination and glycolic acid peels in melasma in Indian patients. J Cosmet Dermatol. 2011;10:68-9.

14. Erbil H, Sezer E, Tastan B, Arca E, Kurumlu Z. Efficacy and safety of serial glycolic acid peels and a topical regimen in the treatment of recalcitrant melasma. J Dermatol. 2007;34:25-30.

15. Saeed W, Altaf F, Rashid S, Rani Z. Efficacy and Safety of $50 \%$ glycolic acid peels in the treatment of melasma in Fitzpatrick's skin type IV and V. J Pak Associat Dermatol. 2016;26:26-30.

16. Bari AU, Iqbal Z, Rehman SB. Superficial chemical peeling with salicylic acid in facial dermatosis. J Coll Physicians Surg Pak. 2007;17:187-90.

Copyright by Uzma Ahsan, et al. This is an open access article distributed under the terms of the Creative Commons Attribution License, which permits unrestricted use, distribution, and reproduction in any medium, provided the original author and source are credited.

Source of Support: Nil, Conflict of Interest: None declared. 